\title{
Ausencia de escenarios deportivos: cambio en las dinámicas socioeconómicas de la población ibaguereña
}

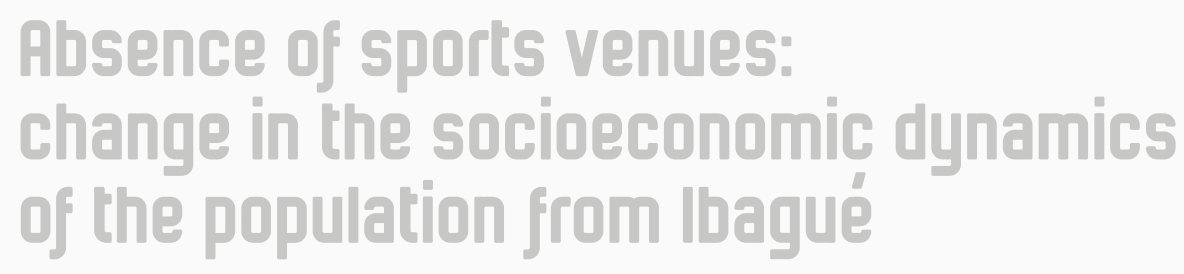

\author{
Oscar Andrés Martínez Torres ${ }^{1}$ \\ Luisa Fernanda Cruz Bustamante?
}

ㅇ.

Recepción: 05/10/2020
(.).

Aprobación: 14/10/2020
(-)

Publicación: 18/12/2020

\section{Para citar este artículo:}

Martínez Torres, O. A., \& Cruz Bustamante, L. F. (2020). Ausencia de escenarios deportivos: cambio en las dinámicas socioeconómicas de la población ibaguereña. Indagare, (8), 69-77. https://doi.org/10.35707/indagare/807

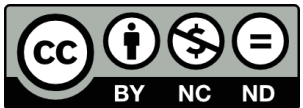

\footnotetext{
1 Grupo de investigación UNIDERE, Universidad de Ibagué, Colombia. Correo electrónico: 1120161001@ estudiantesunibague.edu.co

${ }^{2}$ Grupo de investigación UNIDERE, Universidad de Ibagué, Colombia. Correo electrónico: luisa.cruz@unibague.edu.co
} 


\title{
Resumen
}

Los encuentros nacionales deportivos generan importantes dinámicas socioeconómicas en las ciudades organizadoras, debido a que se vinculan un número significativo de actores de diversos ámbitos: económico, empresarial, capital humano, y el más importante, deportivo. No es un secreto que en Colombia han sucedido un sinfín de inconvenientes jurídicos y de contratación en el desarrollo de estas justas deportivas; sin embargo, lo sucedido en Ibagué marcó un antes y un después desde diversos panoramas. Por consiguiente, esta investigación se centró en la identificación y medición del impacto socioeconómico que generaron los XX Juegos Deportivos Nacionales y los IV Paranacionales 2015 que pasaron de ser una gran oportunidad a dejar sin escenarios deportivos a una ciudad completa.

\begin{abstract}
National sporting events generate significant socio-economic dynamics in host cities, due to the fact that a meaningful number of actors from various fields are linked: economic, business, human capital, and the most important, sports. It is not a secret that countless legal and contracting problems have occurred in Colombia in the development of these sporting events; however, what happened in Ibagué marked a breaking point from different perspectives. Consequently, this research focused on the identification and measurement of the socioeconomic impact generated by the 20th National Games and the 4th Paranational Games 2015, which went from being a great opportunity to leaving an entire city without sport venues.
\end{abstract}

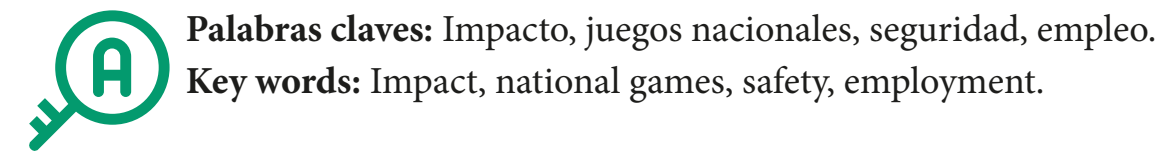

\section{Introducción}

El deporte es una actividad que es parte importante no solo de la recreación de los seres humanos, sino también en el alcance de una vida saludable. La actividad física es todo aquello que compromete el movimiento del cuerpo y hace trabajar de manera coordinada a los músculos. La falta de ella ocasiona un gran número de muertes en el mundo. Estimular el cuerpo trae grandes beneficios para el corazón, los pulmones y los músculos, además al realizarla de manera planificada nos encontramos con el ejercicio físico, pero si se realiza en un medio reglamentado para la competencia, es un deporte. 
La regularidad en la práctica de actividad física trae muchos beneficios para la salud y se recomienda según la edad, que las personas tengan una rutina de ejercicios (Ministerio de Salud, 2015).

La Organización Mundial de la Salud (OMS) describe la actividad física como cualquier movimiento corporal producido por los músculos esqueléticos que exija gasto de energía. La OMS explica que su inactividad es el cuarto factor de riesgo de mortalidad a nivel mundial y es la principal causa de un alto porcentaje de los cánceres de mama y colon, diabetes y cardiopatías. En la sociedad contemporánea los trabajos que la exigen son cada vez más escasos, por eso el deporte cobra cada vez mayor importancia (Clínica Baviera, 2016).

En Colombia, hace 91 años se estableció el evento deportivo abierto más importante a nivel nacional, los Juegos Deportivos Nacionales, que tienen como objetivo congregar los mejores atletas del país e incentivar el crecimiento regional de las ciudades donde se llevan a cabo. Para 2015, los Xx Juegos Deportivos Nacionales y IV Juegos Deportivos Paranacionales tendrían lugar en el departamento del Tolima, con sede principal en Ibagué y apoyo del Chocó. Sin embargo, la construcción y adecuación de los escenarios deportivos estaba sin terminar para el momento en el que se esperaba que las justas iniciaran en Ibagué, lo cual configuró uno de los mayores escándalos en el departamento debido a que las instituciones organizadoras habrían incumplido con lo prometido.

A causa de esto, Ibagué disponía de condiciones impropias para llevar a cabo el evento, hecho que puso a la ciudad en vergüenza nacional además de dejarla sin escenarios donde sus deportistas locales o habitantes en general pudiesen entrenar. Como es de esperarse esta situación ha incidido en los entrenamientos de los atletas ibaguereños de alto rendimiento; no obstante, la ciudadanía, que ha sido espectadora de todo lo ocurrido, era también consumidora y beneficiaria de los servicios prestados por dichas instalaciones antes de esa época, por lo tanto, se deduce que los acontecimientos también generaron efectos en la población.

De las 23 obras por realizarse, 15 serían en el Tolima y 8 en el Chocó. De las 7 adecuaciones, 4 serían en el Tolima y 3 en el Chocó, cifras que significan que el presupuesto en su mayoría se destinaría para el departamento tolimense con sede principal en Ibagué. De acuerdo con datos del Departamento Nacional de Planeación (2014), "las inversiones por realizar para la construcción y adecuación de escenarios deportivos podrán generar aproximadamente 3640 empleos directos, lo que aumenta la generación de ingresos por \$10 000 millones en Chocó y \$20 000 millones en Tolima” (p. 10). 
Una de las más grandes expectativas de cumplir con dichos juegos, es que exista un antes y un después en la región donde se realizan. Según Coldeportes (2017) "las competencias permiten la adecuación y construcción de nuevos escenarios, y la presencia de tantos colombianos reunidos en torno al deporte obliga a los organizadores a arreglar la casa" (s.p.). Por consiguiente, cada ciudad sede se preocupa por la modernización de su entorno, de cara a sectores como el económico, el de infraestructura vial, comunicaciones, turismo, entre otros.

Sin embargo, en Ibagué el antes y después fue muy distinto a lo esperado. Cuando llegó la hora de iniciar el evento deportivo abierto más grande del país, el panorama era desalentador; faltaban 33 días para la inauguración y las obras iban en un 40 \%-50 \% de avance (El Olfato, 2015). Las pocas edificaciones que estaban listas carecían, en su mayoría, de estándares de seguridad. En ese sentido, las disciplinas deportivas se tuvieron que trasladar a otros departamentos y según la Contraloría General de la República, se habrían perdido unos $\$ 66000$ millones.

Esta situación dejó a la capital tolimense sin escenarios deportivos, debido a que aquellos que se esperaban construir quedaron incompletos, y los existentes fueron abandonados a mitad de la remodelación. Como era de esperarse, esto preocupó a deportistas, ligas y funcionarios con cargo dentro de los escenarios, pero la ciudadanía también debía ser tenida en cuenta, pues ha visto el detrimento económico importante para Ibagué, el departamento y la nación, sin contar con las consecuencias en la población (Hoz, 2017).

Esta situación nos llevó a indagar sobre el impacto que generó la problemática en la ciudad en términos socioeconómicos, también, en qué proporción este estudio brinda una aproximación a los impactos generados en diferentes grupos poblacionales de Ibagué ante la ausencia de escenarios deportivos.

\section{Materiales y métodos}

La metodología utilizada para el desarrollo de este proyecto estuvo enfocada en establecer el impacto socioeconómico en Ibagué a partir de las irregularidades en la construcción de los escenarios deportivos para los XX Juegos Deportivos Nacionales y IV Juegos Deportivos Paranacionales 2015. La razón que sustenta lo anterior radica en la necesidad de tener un conocimiento adicional de los argumentos expresados por su comunidad deportiva sobre las debilidades y fortalezas en los sistemas y procesos actuales, a nivel económico, de recurso humano, financieros o de infraestructura. 
La investigación fue de tipo mixto, porque tuvo como características identificar la naturaleza de la realidad, situación que refleja un enfoque cualitativo, pero al momento de agrupar la información y traducirla se refleja un enfoque cuantitativo, que se traduce, finalmente, en la unión de los dos.

Esta investigación es de tipo analítico-descriptivo, porque en ella se parte de la recolección de datos que describen la realidad de los grupos de interés en dos orientaciones: social y económica, datos que permitirán establecer las categorías que determinarían el desarrollo de la investigación para identificar la situación actual. Se ejecutó en Ibagué durante 24 meses y surgió de la priorización de algunos actores que están directamente relacionados con la problemática y contribuyen de forma fundamental al análisis y evaluación de impacto.

La población de la investigación estuvo integrada por seis actores: sector público, sector privado, ciudadanía, habitantes de los sectores afectados, actores sociales y deportistas, para los cuales se crearon indicadores sociales de acuerdo con las categorías de salud, calidad de vida, apoyo social y seguridad, e indicadores de tipo económico relacionados con las categorías de mercado laboral, ingresos ciudadanos, ingresos públicos, ingresos privados, salario, empresas y pensiones. Las técnicas e instrumentos para la recolección de información fueron utilizados según la identificación detallada de los indicadores de impacto que se relacionan con cada uno de los actores priorizados.

\section{Resultados}

Algunos de los resultados relevantes de la investigación corresponden a los relacionados con los indicadores de percepción ciudadana en términos de seguridad y empleo, que se tuvieron en cuenta para cada uno de los actores involucrados en el estudio, por medio de la aplicación de encuestas y entrevistas focalizadas según algunos parámetros particulares de los mismos. En cuanto a los actores que se denominaron habitantes del sector afectado se priorizaron las comunas 4 y 10 que son las que se encuentran ubicadas en el centro de la capital, en especial, en el perímetro que bordea los escenarios deportivos de las piscinas olímpicas y parque deportivo, que son las infraestructuras más amplias destinadas al deporte en la ciudad. De acuerdo con las proyecciones suministradas por la Alcaldía Municipal de Ibagué, para el año 2019 se registran 44077 habitantes en la comuna 4 y 45 149 en la comuna 10.

Por lo tanto, se utilizó la técnica de encuesta a 383 personas habitantes de dichas comunas quienes percibieron un impacto a través del tiempo en categorías como movilidad, seguridad, generación de empleo, entre otras. 
Al consultar a los encuestados sobre el nivel de seguridad en el sector donde se encuentran los escenarios deportivos de la calle 42, 188 (49.09\%) refirieron que la seguridad en esta zona era buena. Durante la realización de los juegos, 59 (15.40\%) indicaron que así lo seguía siendo; sin embargo, después de realizarse, solo 20 apuntaron lo mismo. Por otra parte, antes de los juegos, 46 encuestados (12.01\%) refirieron que la seguridad en la zona era mala, durante el cumplimiento de las justas este indicador incrementó a 119 personas (31.07 \%) y después de ellas, a 156 (40.73 \%). Esto arrojó una variación del 239 \% entre el antes y el después de la seguridad en la zona descrita con anterioridad.

Figura 1. Percepción ciudadana sobre el nivel de seguridad en el sector

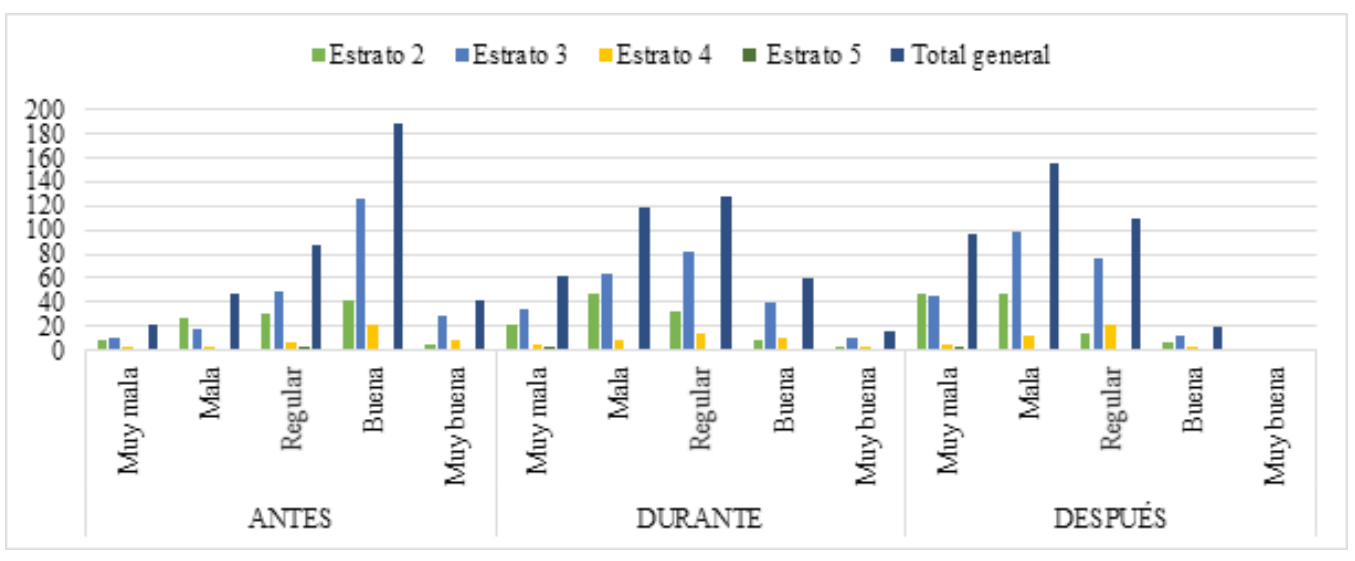

Fuente: elaboración propia

Por último, respecto de la generación de empleo en la ciudad, solo 47 personas encuestadas (12.27 \%) manifestaron que la generación de empleo en Ibagué antes de los juegos era buena, durante las justas, 36 (9.40\%) refirieron que era buena y, después de los juegos, 30 personas ( $7.83 \%$ ) manifestaron que seguía siendo buena. Asimismo, 120 encuestados (31.33\%) respondieron que antes de los juegos la generación de empleo era regular, esta percepción incrementó durante su desarrollo, puesto que, 150 personas (39.16 \%) dijo que era regular y después de los juegos, 159 (41.51\%) expresaron que era regular. Esto constató una variación de $33 \%$ entre el antes y el después en la percepción de la generación de empleo en Ibagué. 
INDAGAZEE e-ISSN: 2357-5042 • Número 8 (2020) • Universidad de Ibagué • doi: https://doi.org/10.35707/indagare/807

Figura 2. Percepción ciudadana sobre la generación de empleo en la ciudad

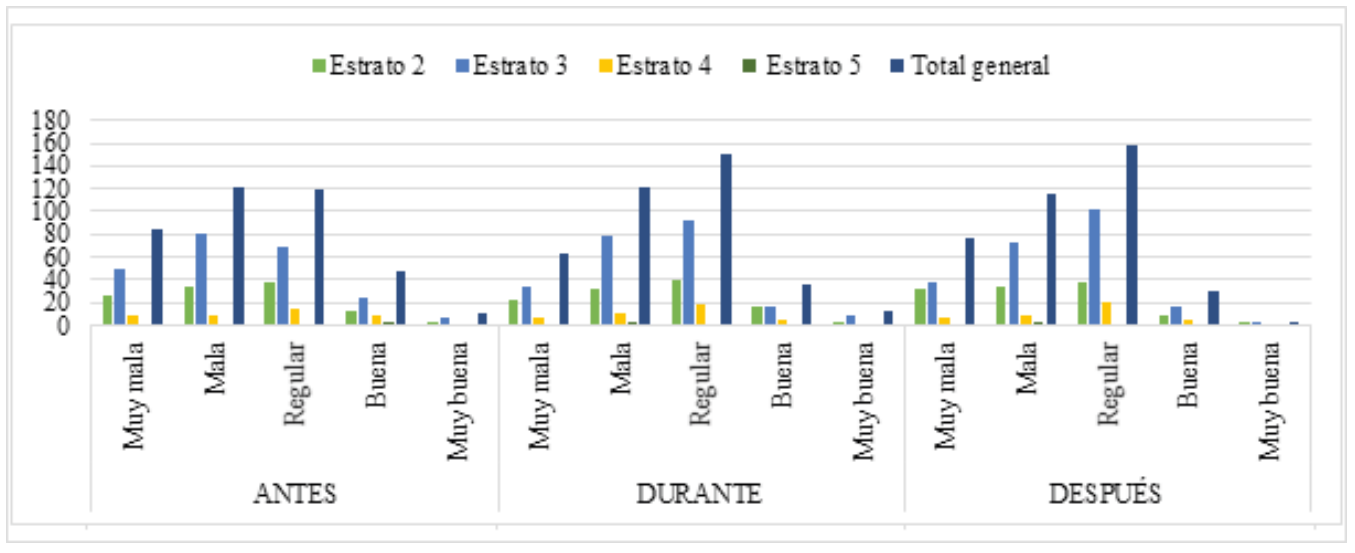

Fuente: elaboración propia

\section{Potencial uso}

Los resultados del presente estudio favorecen a los ibaguereños porque muestran el impacto generado por las irregularidades en la construcción de los escenarios deportivos en la ciudad, además, de la afectación en aspectos como seguridad y generación de empleo. Estos temas pueden ser utilizados para futuras investigaciones de mayor profundidad, debido a que la experiencia obtenida con la metodología utilizada permite que se identifiquen los impactos socioeconómicos de acuerdo con varias categorías de análisis.

Entre los principales hallazgos se encontró una gran variación frente a la percepción de las personas antes, durante y después de los juegos en materia de escenarios deportivos. Un ejemplo claro es el coliseo cubierto que antes de los juegos, el $59.27 \%$ de los encuestados indicaba que se encontraba en buenas condiciones, durante los juegos solo el 25.59 \% conservaba la misma percepción y después, tan solo el 14.36 \% seguía pensando lo mismo. Estos porcentajes arrojaron una variación negativa del $75.77 \%$ entre el antes y el después de las justas. Lo anterior devela que los habitantes del sector sí se vieron impactados de forma negativa por las irregularidades de la construcción de los escenarios deportivos durante el pasar del tiempo.

En materia de seguridad, los habitantes del sector expresaron que este indicador cambió de manera significativa entre el antes, durante y después de los juegos. Por ejemplo, antes, el $49.09 \%$ de las personas manifestaban que la seguridad era buena en la zona; durante los juegos, este indicador quedó en 15.40 \% y después cayó al $5.22 \%$. Esto arrojó una variación negativa del $89 \%$. 
Lo obtenido en esta investigación se respalda con las estadísticas consolidadas por el Centro de Información Municipal Para la Planeación Participativa (CIMPP) (2019), puesto que presenta que el número de hurtos aumentó gradualmente desde 2015 a 2018. En 2015 hubo en total 3498 hurtos y para el año siguiente 4 330. Esto demuestra un incremento del $23.78 \%$. Por otra parte, para 2017 los hurtos fueron 5313 en toda la ciudad, esto muestra un aumento de 2016 a 2017 del $22.70 \%$. Asimismo, el hurto pasó de 5313 a 5866 para 2018 y arrojó un incremento del $10.4 \%$ de ese año con respecto al anterior.

Por último, la generación de empleo en Ibagué fue un punto clave que se trabajó con las personas encuestadas, debido a que la ciudad siempre ha estado por encima del promedio de tasa de desempleo a nivel nacional y es importante conocer el punto de vista de los habitantes del sector frente a este ítem. Del total de los encuestados, el $12.27 \%$ indicó que la generación de empleo en la ciudad antes de los juegos era buena, durante su celebración la percepción seguía siendo buena con el 9.40 \% y después esta percepción terminó en $7.83 \%$, lo cual estableció como resultado una variación negativa del $36 \%$ entre el antes y el después del evento. Asimismo, el $31.33 \%$ de los encuestados probó que la generación de empleo en la capital del Tolima era regular antes de su celebración. Durante su desarrollo esta percepción llegó al 39.16 \% y después quedó en el $41.51 \%$, porcentajes que manifestaron una variación del $33 \%$ entre el antes y después.

\section{Ficha técnica del proyecto}

Título del proyecto: Evaluación del impacto socioeconómico de las irregularidades en la construcción de los escenarios para los XX Juegos Nacionales 2015 en la ciudad de Ibagué. PRIT: Desarrollo regional inclusivo y sustentable.

Código del proyecto: $18-564-$ INT.

Palabras claves: Juegos nacionales, escenarios deportivos, Ibagué, impacto socioeconómico.

Grupo de investigación: UNIDERE.

Investigador principal: Carlos Alberto Salazar Villalba.

Correo electrónico: carlos.salazar@unibague.edu.co 
INDAGA]ZE e-ISSN: 2357-5042 • Número 8 (2020) • Universidad de Ibagué • doi: https://doi.org/10.35707/indagare/807

\section{Referencias}

Clínica Baviera (13 de junio de 2016). La importancia del deporte para una vida saludable. Recuperado de https://n9.cl/ap424

Centro de Información Municipal para la Planeación Participativa. (2019). Anuario estadístico municipal. Ibagué 2018-2019. Recuperado de https://n9.cl/dh59

Coldeportes. (2017). Juegos Deportivos Nacionales y Juegos Deportivos Paranacionales. Recuperado de https://bit.ly/38hgOfg

Departamento Nacional de Planeación. (2014). Gobierno nacional aprueba inversión por \$215.250 millones para escenarios deportivos en Tolima y Chocó. Recuperado de https://bit.ly/33Ad3jl

El Olfato. (05 de octubre de 2015). Ibagué, protagonista del ¡Qué tal esto! de Noticias UNO por las obras de los Juegos Nacionales. El Olfato. Recuperado de https://bit.ly/3fVfTo9

Hoz, J. M. (24 de septiembre de 2017). El olvido de los escenarios deportivos de Ibagué. El Espectador. Recuperado de https://n9.cl/wmwvl

Ministerio de Salud. (2015). ABECÉ. Actividad física para la salud. Recupreado de https://n9.cl/e389o 